\title{
A phase I dose-finding study based on polychotomous toxicity responses
}

\author{
XiaObin Yang and Keying Ye Y $^{* \dagger}$
}

In phase I clinical trials, toxicity study is important and the toxicity level is often categorized into multiple (polychotomous) grades, rather than dichotomous grades. In this paper, we introduce a concept of overall maximum tolerated dose (overall MTD) and we discuss its analytic properties. The traditional definition of the MTD is shown to be a special case of the overall MTD. A dose-finding strategy is also proposed to obtain the overall MTD. Motivated by the continual reassessment method (CRM), a cumulative probit model with latent variables is introduced to fit the data. By introducing latent variables, Markov chain Monte Carlo (MCMC) methods are employed to estimate the model parameters. Simulation studies show that the cumulative probit model, which takes into account the severity level of toxicity, reduces the number of patients allocated to the higher toxicity dose level. This could reduce the risk of toxicity for patients in the phase I study.

AMS 2000 SUBJECT ClASSifications: Primary 62F15, 62L05; secondary 62P10.

Keywords AND Phrases: Phase I clinical trial, Polychotomous toxicity responses, Dose finding, Continual reassessment method (CRM), Cumulative probit model, Latent variable, Markov chain Monte Carlo (MCMC).

\section{INTRODUCTION}

The primary goal of a phase I clinical trial is to determine the dose of a candidate drug to use in a subsequent phase II trial. Issues of side effects due to toxicity in the drug, or the dose limiting toxicity (DLT) is always a main concern in phase I study. Toxicity level is often categorized into multiple grades. For instance, the general guidelines of the Common Toxicity Criteria (CTC) (National Cancer Institute, 2003) are grade 0 for no toxicity; grade 1, 2, 3, 4 and 5 for minimal toxicity, moderate toxicity, severe toxicity, life threatening and death, respectively. In most dose allocation procedures, such as the traditional " $3+3$ " design [7], CRM [6] and EWOC [2], these grades are dichotomized. For example, if grade 4 fatigue is considered DLT then grades $0-3$ will be non-DLT and treated identically from the point of

* Corresponding author.

${ }^{\dagger}$ Dr. Keying Ye's research was partially supported by a grant from the College of Business at the University of Texas at San Antonio. view of an experimental design. Such dichotomization works for relatively mild toxicities. However, for severe and possibly irreversible effects such as renal, liver, or neurological toxicities, grade 4 renal toxicity is much more dangerous than that for grade 3 . Hence, those toxicity grades cannot be treated equivalently. Such concerns need be addressed in the dose escalation process.

In 2009, Yin and Yuan [10] proposed using multiple parallel CRM models, each with a different set of prespecified toxicity probabilities. In the Bayesian paradigm, they assign a discrete probability mass to each CRM model as the prior model probability. The posterior probabilities of toxicity can be estimated by the Bayesian model averaging (BMA) approach. Dose escalation or deescalation is determined by comparing the target toxicity rate and the BMA estimates of the dose toxicity probabilities. Yin and Yuan examine the properties of the BMA-CRM approach through extensive simulation studies, and also compare this new method and its variants with the original CRM. The results demonstrate that the BMA-CRM is competitive and robust, and eliminates the arbitrariness of the prespecification of toxicity probabilities. Unfortunately, the BMA-CRM approach dose not take the multiple toxicity grade level into account.

During the past ten years, polychotomous toxicity response has been widely discussed. In 2000, Wang et al. [8] extended the CRM by incorporating the idea of unequal weights on the assessments of grade 3 and grade 4 toxicity in the dose escalation. The simulation results show that their procedures reduce the chance of recommendation to the higher dose levels by taking into account the impact of grade 4 toxicity, both for the standard design and for the CRM. Similar trends are observed for patient allocation to the higher levels. Additionally, for CRM which performs more accurately on the estimation of maximum tolerated dose (MTD), the proposed extended CRM maintains the same characteristic.

In 2004, Bekele and Thall [3] proposed a Bayesian method for dose finding in a sarcoma trial based on a vector of correlated, ordinal-valued toxicities with severity levels varying with dose. They also developed a method for jointly eliciting the prior, a vector of weights quantifying the clinical importance of each level of each type of toxicity, and a target total toxicity burden (TTB) acceptable to the physicians.

There is other research related to this type of problem. In 2007, Yuan et al. [11] proposed another extension of the continual reassessment method (CRM), called the Quasi-CRM, 
to incorporate the grade information. They convert the toxicity grades to numeric scores that reflect their impacts on the dose allocation procedure, and then incorporate those scores into the CRM using the quasi-Bernoulli likelihood. A simulation study demonstrates that the Quasi-CRM is superior to the standard CRM and comparable to a univariate version of the Bekele and Thall method [3].

In Phase I clinical trial, maximum tolerated dose (MTD) refers to the highest dose of a drug or treatment that does not cause unacceptable side effects and the MTD is determined in clinical trials by testing increasing doses on different groups of people until the highest dose with acceptable side effects is found (Dictionary of Cancer Terms. National Cancer Institute). This is a descriptive definition rather than an analytical definition, and it leads to different interpretations and comprehension of MTD in practice.

For most of the model-based designs, MTD is defined as a dose, $x^{*}$, at which the probability of DLT is equal to $\theta$, where $\theta$ is an aimed-for target DLT probability. This definition of MTD works well under the case of dichotomous toxicity responses. However, it is difficult to directly apply such a definition in the case of polychotomous toxicity responses. For example, suppose the probability configurations for toxicity grades $0-5$ at dose level $x_{1}$ and $x_{2}$ are $p_{1}=(0.10,0.25,0.35,0.15,0.10,0.05)$ and $p_{2}=(0.35,0.25,0.10,0.05,0.10,0.15)$, respectively, where the $p_{i j}$ is the probability that the patient suffers the $j$ th toxicity grade at dose $x_{i}, j=1, \ldots, 6, i=1,2$. For instance, $p_{13}=0.35$ means the chance that the patient suffers a $3 \mathrm{rd}$ toxicity grade at dose $x_{1}$ is 35 percent. Assume that there is a DLT if the toxicity grade is 4 or above and non-DLT otherwise and set the target DLT probability $\theta$ equals to 0.30 . Since $P\left(\mathrm{DLT} \mid x_{1}\right)=P\left(\mathrm{DLT} \mid x_{2}\right)=0.30$, both dose levels $x_{1}$ and $x_{2}$ are MTD according to such definition, i.e. there is no difference between dose levels $x_{1}$ and $x_{2}$ in the sense of the probability of DLT. It is obvious that these two dose levels are not the same by comparing their probability configurations. For dose $x_{1}$, the probability mass concentrates at a third toxicity grade with probability 0.35 . However, for dose $x_{2}$, the probability mass has dispersed concentrations at first and sixth toxicity grades with probabilities 0.35 and 0.15 , respectively. Hence, it is hard to generate the definition of the MTD from the case of dichotomous toxicity responses to that of the polychotomous toxicity responses naturally.

In the case of polychotomous toxicity responses, Bekele and Thall [3] define MTD as the dose at which the total toxicity burden (TTB) is equal to a target TTB. A numerical variable, so called severity weight, is defined on the ordered toxicity grade. Then the TTB is obtained by calculating the mean value of the severity weight. Simulation study shows that, on average, this definition performs well under a wide variety of circumstances. However, in practice, the meaning of the target TTB is not quite straightforward (e.g., 3.04 in Bekele and Thall's example [3]) and it requires a great deal of effort to interact with the physicians for establishing severity weight as well as target TTB.
It is thus important to reconsider the definition of MTD, which can be applied in both cases of dichotomous and polychotomous toxicity responses and meanwhile can be easily interpreted by physicians. In this research, we attempt to give a more rigorous definition for MTD and we also propose an attractable way to deal with the computation of the MTD. In Yang, Ye and Wang, 2011 [9] a probit model with latent variables in the case of dichotomous toxicity responses is studied. In this article, a cumulative probit model with latent variables will be investigated in the case of polychotomous toxicity responses. In the next section, we introduce the new definition of overall MTD along with its analytic properties. In Section 3, the cumulative probit model with latent variables and the full conditional distributions are given. The likelihood function and the posterior distribution functions of the model parameters will be given in Section 4. In Section 5, a simulation study exploring operating characteristics of the proposed method is presented. Finally, in Section 6, conclusions and discussions are provided.

\section{THE OVERALL MTD}

Suppose that, instead of a binary definition of toxicity, we use an $M$-point ordinal toxicity scale. Denoted by $Y$ the polychotomous toxicity response which takes one of the $M$ values, $\{1, \ldots, M\}$. Define by $p_{y}(x)$ the probability of suffering toxicity grade $Y=y, y=1, \ldots, M$, at dose $x$ such that $\sum_{y=1}^{M} p_{y}(x)=1$ for all $x \in \mathbb{X}$ (where $\mathbb{X}$ is the set of all interesting dose levels). Set a critical toxicity grade $m, m \in$ $\{1,2, \ldots, M\}$, such that there is a severe toxicity response if the toxicity grade is $m$ or higher, or, simply $Y \geq m$, not a severe toxicity response if the toxicity grade is less than $m$, or $Y<m$. Such severe toxicity is named as level- $m$ severe toxicity, denoted by $T_{m}=\{Y \geq m\}$. For any level$m$ severe toxicity $T_{m}$, there is a target toxicity probability $\theta_{m}, m \in\{1,2, \ldots, M\}$. A dose $x$ is said level- $\boldsymbol{m}$ tolerable if the probability of level- $m$ severe toxicity $T_{m}$ at $x$ is less than or equal to $\theta_{m}$, i.e. $P(Y \geq m \mid x) \leq \theta_{m}$, not level- $\boldsymbol{m}$ tolerable if the probability of $T_{m}$ at $x$ is greater than $\theta_{m}$, i.e. $P(Y \geq m \mid x)>\theta_{m}$. Given a critical toxicity grade $m$, the maximum dose of all level- $m$ tolerable doses is called the level- $m$ maximum tolerated dose. To make it clearer, we introduce the following definition.

Definition 2.1. For critical toxicity grade $m, m \in$ $\{1,2, \ldots, M\}$, associated with its target severe toxicity probability $\theta$, the level- $\boldsymbol{m}$ maximum tolerated dose (or briefly level- $m$ MTD), denoted by ${ }_{m} \operatorname{MTD}(\theta)$, is defined as

$$
\begin{aligned}
{ }_{m} \operatorname{MTD}(\theta) & =\sup \{x \mid P(Y \geq m \mid x) \leq \theta\} \\
& =\sup \left\{x \mid \sum_{y=m}^{M} p_{y}(x) \leq \theta\right\},
\end{aligned}
$$

where $\sup \{S\}$ is the supremum of set $S$. 


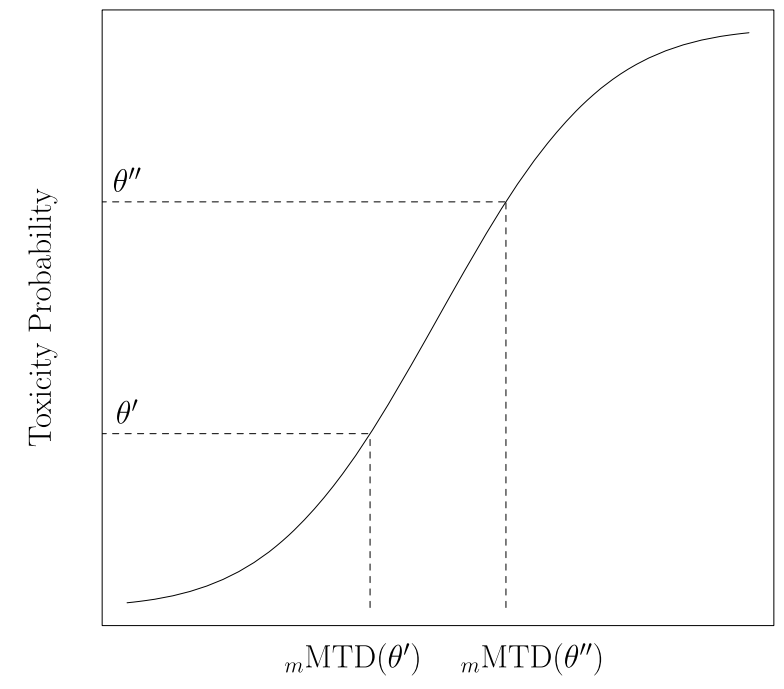

Figure 1. Graph illustration for Proposition 2.1. It shows that the level-m MTD, ${ }_{m} M T D(\theta)$, is increasing in its corresponding target toxicity probability $\theta$.

Here, we take the supremum because of the belief that, given a target toxicity probability, the higher the dosage, the more efficient the chemical compound, i.e. it is assumed that dose-response curves for both toxicity and efficacy are increasing in the dosage, or, simply expressed, "the more pain, the more gain."

The following propositions show some properties of the level- $m$ MTD.

Proposition 2.1. For any critical toxicity grade $m, m \in$ $\{1,2, \ldots, M\},{ }_{m} \operatorname{MTD}(\theta)$ is increasing in $\theta$, where $\theta$ is the target toxicity probability associated with the critical toxicity grade $m$ (see Figure 1).

Proof. Suppose $\theta^{\prime}<\theta^{\prime \prime}$, then $\sum_{y=m}^{M} p_{y}(x) \leq \theta^{\prime}$ implies $\sum_{y=m}^{M} p_{y}(x) \leq \theta^{\prime \prime}$, for $m \in\{1,2, \ldots, M\}$. Hence, $\{x \mid$ $\left.\sum_{y=m}^{M} p_{y}(x) \leq \theta^{\prime}\right\} \subset\left\{x \mid \sum_{y=m}^{M} p_{y}(x) \leq \theta^{\prime \prime}\right\}$. Therefore,

$$
\begin{aligned}
{ }_{m} \operatorname{MTD}\left(\theta^{\prime}\right) & =\sup \left\{x \mid \sum_{y=m}^{M} p_{y}(x) \leq \theta^{\prime}\right\} \\
& \leq \sup \left\{x \mid \sum_{y=m}^{M} p_{y}(x) \leq \theta^{\prime \prime}\right\} \\
& ={ }_{m} \operatorname{MTD}\left(\theta^{\prime \prime}\right) .
\end{aligned}
$$

Hence, ${ }_{m} \operatorname{MTD}(\theta)$ is increasing in $\theta$.

Referring to any level- $m$ maximum tolerated dose, ${ }_{m} \operatorname{MTD}(\theta)$, Proposition 2.1 shows the higher the tolerance probability $\theta$, the larger the amount of the dose should be applied to achieve the maximum efficacy of the drug. The following proposition shows that, on the other side, the

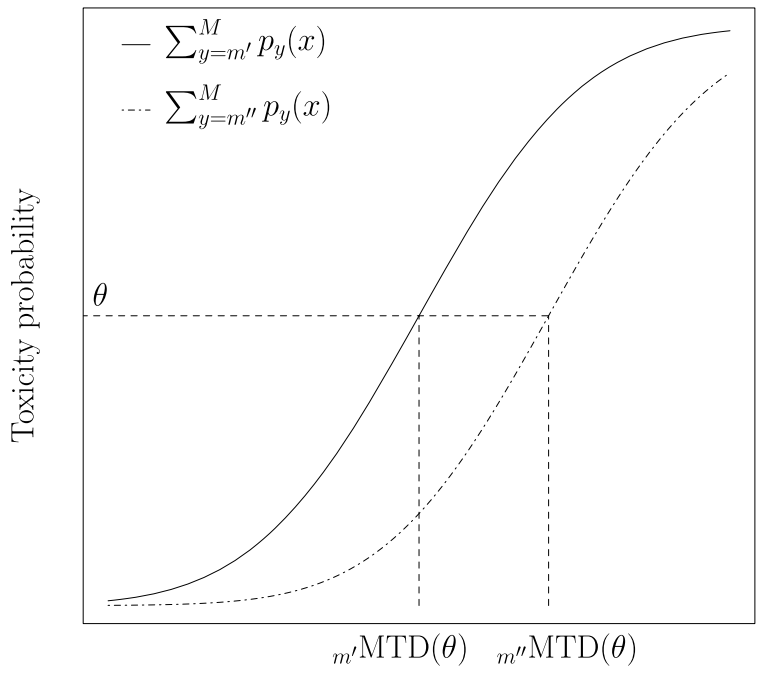

Figure 2. Graph illustration for Proposition 2.2. It shows that, for a fixed target toxicity probability $\theta,{ }_{m} M T D(\theta)$ is increasing in the toxicity grade $m$.

higher the toxicity grade which is treated as the DLT, the larger the amount of the drug that can be tolerated.

Proposition 2.2. Given a target toxicity probability $\theta$, ${ }_{m} \operatorname{MTD}(\theta)$ is increasing in toxicity grade $m$, i.e., $m^{\prime} \operatorname{MTD}(\theta) \leq_{m^{\prime \prime}} \operatorname{MTD}(\theta)$, for any $m^{\prime}<m^{\prime \prime}$ in $\{1,2, \ldots, m\}$ (see Figure 2).

Proof. Suppose $m^{\prime}<m^{\prime \prime}$, then $\sum_{y=m^{\prime}}^{M} p_{y}(x) \geq \sum_{y=m^{\prime \prime}}^{M} p_{y}(x)$, Hence,

$$
\left\{x \mid \sum_{y=m^{\prime}}^{M} p_{y}(x) \leq \theta\right\} \subset\left\{x \mid \sum_{y=m^{\prime \prime}}^{M} p_{y}(x) \leq \theta\right\} .
$$

Therefore,

$$
\begin{aligned}
m^{\prime} \operatorname{MTD}\left(\theta^{\prime}\right) & =\sup \left\{x \mid \sum_{y=m^{\prime}}^{M} p_{y}(x) \leq \theta\right\} \\
& \leq \sup \left\{x \mid \sum_{y=m^{\prime \prime}}^{M} p_{y}(x) \leq \theta\right\} \\
& ={ }_{m^{\prime \prime}} \operatorname{MTD}(\theta) .
\end{aligned}
$$

Hence, ${ }_{m} \operatorname{MTD}(\theta)$ is increasing in $m$.

In order to define the overall MTD in the case of polychotomous toxicity responses with $M$-point ordinal toxicity grade, let us introduce the following notations. Denoted by $\mathbb{Y}=\{1, \ldots, M\}$ the set of ordered grades that are associated with the polychotomous toxicity response $Y$. Suppose $\boldsymbol{P}=\left\{p_{1}(x), \ldots, p_{M}(x) \mid x \in \mathbb{X}\right\}$ such that $\sum_{y=1}^{M} p_{y}(x)=1$, for all $x \in \mathbb{X}$, and $\boldsymbol{\theta}=\left\{\theta_{1}, \ldots, \theta_{M}\right\}$, where $\theta_{m}$ is the target 


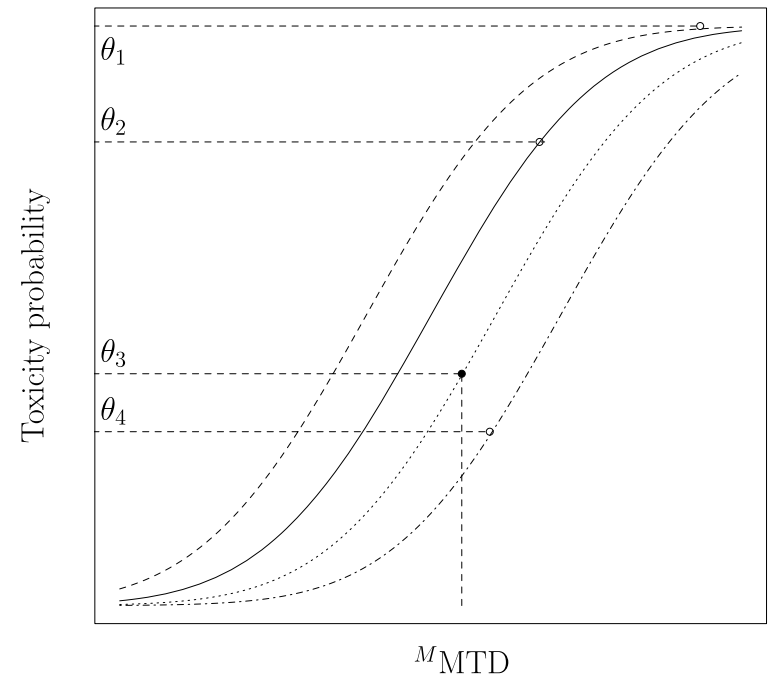

Figure 3. Graph illustration for Definition 2.2. In this graph, there are 4 increasing curves

$P\left(Y \geq m_{i} \mid x\right)=\sum_{y=m_{i}}^{M} p_{y}(x), i=1, \ldots, 4$, where

$m_{1}<\cdots<m_{4}$ are some toxicity grades. There is a level- $m_{i}$

MTD given the target toxicity probability $\theta_{i}$, denoted by a

small circle, $i=1, \ldots, 4$. The overall MTD, denoted by a

dotted circle, is the minimum of all level-mi MTD's.

toxicity probability corresponding to the level- $m$ severe toxicity $T_{m}, m \in\{1,2, \ldots, M\}$. The triplet $\{\mathbb{Y}, \boldsymbol{P}, \boldsymbol{\theta}\}$ is called an $\boldsymbol{M}$-point ordinal toxicity grade system defined on $\mathbb{X}$.

Example 2.1. Suppose $\mathbb{Y}=\{1,2\}$, where 1 indicates a non-DLT and 2 a DLT. Let $\boldsymbol{P}=\{1-p(x), p(x) \mid x \in \mathbb{X}\}$ and $\boldsymbol{\theta}=\{1, \theta\}$, then $\{\mathbb{Y}, \boldsymbol{P}, \boldsymbol{\theta}\}$ is a 2-point ordinal toxicity grade system, or dichotomous toxicity grade system, defined on $\mathbb{X}$. Furthermore, if $p(x)=[(1+\tanh x) / 2]^{a}$ and $\theta=0.2$, then the original CRM by O'Quigley et al. [6] can be reconsidered under this framework $\{\mathbb{Y}, \boldsymbol{P}, \boldsymbol{\theta}\}$.

Suppose $\{\mathbb{Y}, \boldsymbol{P}, \boldsymbol{\theta}\}$ is an $M$-point ordinal toxicity grade system defined on $\mathbb{X}$. Based on Definition 2.1, dose $x$ is level$m$ tolerable if and only if dose $x \leq{ }_{m} \operatorname{MTD}\left(\theta_{m}\right)$. Hence, the overall MTD of $\{\mathbb{Y}, \boldsymbol{P}, \boldsymbol{\theta}\}$ should be defined as the minimum of all the level- $m$ MTD. To make it clearer, we introduce the following definition.

Definition 2.2. Given $M$ dimensional vector $\boldsymbol{\theta}=$ $\left(\theta_{1}, \theta_{2}, \ldots, \theta_{M}\right)$, whose elements $\theta_{m}$ 's are pre-specified target toxicity probabilities with respect to the critical toxicity grade $m$ 's, the overall MTD of the $M$-point ordinal toxicity grade system, denoted by ${ }^{M} \mathbf{M T D}(\boldsymbol{\theta})$, is defined as

$$
{ }^{M} \operatorname{MTD}(\boldsymbol{\theta})=\min \left\{{ }_{m} \operatorname{MTD}\left(\theta_{m}\right) \mid m=1,2, \ldots, M\right\},
$$

where ${ }_{m} \operatorname{MTD}\left(\theta_{m}\right)$ is the level- $m$ MTD associated with its target toxicity probability $\theta_{m}$, for $m \in\{1,2, \ldots, M\}$ (see Figure 3).

454 X. Yang and K. Ye
The following theorem shows that the target toxicity probability $\theta_{m}$ 's should be monotone decreasing in practice, i.e., $\theta_{1}>\theta_{2}>\cdots>\theta_{M}$. Otherwise, the toxicity grade system can be reduced to a lower dimension system.

Theorem 2.1. Suppose $\{\mathbb{Y}, \mathbf{P}, \boldsymbol{\theta}\}$ is an $M$-point ordinal toxicity grade system with its associated target toxicity probability $\boldsymbol{\theta}=\left(\theta_{1}, \theta_{2}, \ldots, \theta_{M}\right)$ and $\mathbf{P}=\left\{p_{1}(x), \ldots\right.$, $\left.p_{M}(x) \mid x \in \mathbb{X}\right\}$. If there exists an $m_{0}$ such that $\theta_{m_{0}} \geq$ $\theta_{m_{0}-1}$, then the $M$-point ordinal toxicity grade system $\{\mathbb{Y}, \mathbf{P}, \boldsymbol{\theta}\}$ is equivalent to the $(M-1)$-point ordinal toxicity grade system $\left\{\mathbb{Y}^{*}, \mathbf{P}^{*}, \boldsymbol{\theta}^{*}\right\}$, where $\mathbb{Y}^{*}=$ $\{1, \ldots, M-1\}, \mathbf{P}^{*}=\left\{p_{1}(x), \ldots, p_{m_{0}-2}(x), p_{m_{0}-1}(x)+\right.$ $\left.p_{m_{0}}(x), p_{m_{0}+1}(x), \ldots, p_{M}(x) \mid x \in \mathbb{X}\right\}$ and $\boldsymbol{\theta}^{*}=\left\{\theta_{1}, \ldots\right.$, $\left.\theta_{m_{0}-1}, \theta_{m_{0}+1}, \ldots, \theta_{M}\right\}$. Hence, the toxicity grade $m_{0}-1$ and $m_{0}$ should be combined into one single toxicity grade level $m_{0}-1$. Here, "equivalence" is in the sense of finding the overall MTD.

Proof. Denoted by ${ }^{M} \operatorname{MTD}(\boldsymbol{\theta})$ and ${ }^{M-1} \operatorname{MTD}^{*}\left(\boldsymbol{\theta}^{*}\right)$ the overall MTD of $\{\mathbb{Y}, \boldsymbol{P}, \boldsymbol{\theta}\}$ and $\left\{\mathbb{Y}^{*}, \boldsymbol{P}^{*}, \boldsymbol{\theta}^{*}\right\}$, respectively. Since $\theta_{m_{0}} \geq \theta_{m_{0}-1}$, using Propositions 2.1 and 2.2, we have

$$
m_{0}-1 \operatorname{MTD}\left(\theta_{m_{0}-1}\right) \leq m_{0} \operatorname{MTD}\left(\theta_{m_{0}-1}\right) \leq m_{0} \operatorname{MTD}\left(\theta_{m_{0}}\right),
$$

where ${ }_{m} \operatorname{MTD}(\theta)$ is the level- $m$ MTD associated with system $\{\mathbb{Y}, \boldsymbol{P}, \boldsymbol{\theta}\}$. Hence,

$$
\begin{aligned}
{ }^{M} \operatorname{MTD}(\boldsymbol{\theta})= & \min \left\{{ }_{m} \operatorname{MTD}\left(\theta_{m}\right) \mid m=1, \ldots, M\right\} \\
= & \min \left\{{ }_{m} \operatorname{MTD}\left(\theta_{m}\right) \mid m=1, \ldots,\right. \\
& \left.m_{0}-1, m_{0}+1, \ldots, M\right\} .
\end{aligned}
$$

Since $\boldsymbol{P}^{*}=\left\{p_{1}(x), \ldots, p_{m_{0}-2}(x), p_{m_{0}-1}(x)+p_{m_{0}}(x)\right.$, $\left.p_{m_{0}+1}(x), \ldots, p_{M}(x) \mid x \in \mathbb{X}\right\}$, one has $\sum_{y=m}^{M-1} p_{y}^{*}(x)=$ $\sum_{y=m}^{M} p_{y}(x)$ for $m \leq m_{0}-1$ and $\sum_{y=m}^{M-1} p_{y}^{*}(x)=$ $\sum_{y=m+1}^{M} p_{y}(x)$ for $m>m_{0}-1$. Furthermore, since $\boldsymbol{\theta}^{*}=$ $\left\{\theta_{1}, \ldots, \theta_{m_{0}-1}, \theta_{m_{0}+1}, \ldots, \theta_{M}\right\}$, one has $\theta_{m}^{*}=\theta_{m}$ for $m \leq$ $m_{0}-1$ and $\theta_{m}^{*}=\theta_{m+1}$ for $m>m_{0}-1$. Consequently, $\left\{\sum_{y=m}^{M-1} p_{y}^{*}(x) \leq \theta_{m}^{*}\right\}$ is equivalent to $\left\{\sum_{y=m}^{M} p_{y}(x) \leq \theta_{m}\right\}$ for $m \leq m_{0}-1$, and $\left\{\sum_{y=m}^{M-1} p_{y}^{*}(x) \leq \theta_{m}^{*}\right\}$ is equivalent to $\left\{\sum_{y=m+1}^{M} p_{y}(x) \leq \theta_{m+1}\right\}$ for $m>m_{0}-1$.

Therefore, according to (1), ${ }_{m} \operatorname{MTD}^{*}\left(\theta_{m}^{*}\right)={ }_{m} \operatorname{MTD}\left(\theta_{m}\right)$ for $m \leq m_{0}-1$ and ${ }_{m} \operatorname{MTD}^{*}\left(\theta_{m}^{*}\right)={ }_{m+1} \operatorname{MTD}\left(\theta_{m+1}\right)$ for $m>m_{0}-1$, where ${ }_{m} \operatorname{MTD}^{*}\left(\theta_{m}^{*}\right)$ is the level- $m$ MTD associated with system $\left\{\mathbb{Y}^{*}, \boldsymbol{P}^{*}, \boldsymbol{\theta}^{*}\right\}$. Finally, using (3),

$$
\begin{aligned}
{ }^{M} \operatorname{MTD}(\boldsymbol{\theta})= & \min \left\{{ }_{m} \operatorname{MTD}\left(\theta_{m}\right) \mid m=1, \ldots, m_{0}-1,\right. \\
& \left.m_{0}+1, \ldots, M\right\} \\
= & \min \left\{{ }_{m} \operatorname{MTD}^{*}\left(\theta_{m}^{*}\right) \mid m=1, \ldots, M-1\right\} \\
= & { }^{M-1} \operatorname{MTD}^{*}\left(\boldsymbol{\theta}^{*}\right),
\end{aligned}
$$

which implies that $\{\mathbb{Y}, \boldsymbol{P}, \boldsymbol{\theta}\}$ is equivalent to $\left\{\mathbb{Y}^{*}, \boldsymbol{P}^{*}, \boldsymbol{\theta}^{*}\right\}$ in the sense of finding the overall MTD.

Repeatedly applying Theorem 2.1, we obtain the following corollary. 
Corollary 2.1. Suppose $\{\mathbb{Y}, \mathbf{P}, \boldsymbol{\theta}\}$ is an $M$-point ordinal toxicity grade system with its associated target toxicity probability $\boldsymbol{\theta}=\left(\theta_{1}, \theta_{2}, \ldots, \theta_{M}\right)$ and $\mathbf{P}=\left\{p_{1}(x), \ldots, p_{M}(x) \mid x \in\right.$ $\mathbb{X}\}$. If there exists an $m_{0}$ such that $\theta_{m}=1$, for $m<m_{0}$ and $\theta_{m}=\theta<1$, for $m \geq m_{0}$, then the $M$-point ordinal toxicity grade system $\{\mathbb{Y}, \mathbf{P}, \boldsymbol{\theta}\}$ is equivalent to the 2-point ordinal toxicity grade system $\left\{\mathbb{Y}^{*}, \mathbf{P}^{*}, \boldsymbol{\theta}^{*}\right\}$, where $\mathbb{Y}^{*}=\{1,2\}$, $\mathbf{P}^{*}=\left\{\sum_{y=1}^{m_{0}-1} p_{y}(x), \sum_{y=m_{0}}^{M} p_{y}(x) \mid x \in \mathbb{X}\right\}$ and $\boldsymbol{\theta}^{*}=\{1, \theta\}$. Furthermore,

$$
{ }^{M} \operatorname{MTD}(\boldsymbol{\theta})={ }^{2} \operatorname{MTD}^{*}\left(\boldsymbol{\theta}^{*}\right)=\sup \left\{x \mid \sum_{y=m_{0}}^{M} p_{y}(x) \leq \theta\right\} .
$$

So, for the admissibility requirement, we assume $1=\theta_{1}>$ $\theta_{2}>\cdots>\theta_{M}>0$. Since $\theta_{1}=1$ and $\sum_{y=1}^{M} p_{y}(x)=1$, ${ }_{1} \operatorname{MTD}(1)=\sup \left\{x \mid \sum_{y=1}^{M} p_{y}(x) \leq 1\right\}=\sup \{\mathbb{X}\}$. Hence, (2) is equivalent to

$$
{ }^{M} \operatorname{MTD}(\boldsymbol{\theta})=\min \left\{{ }_{m} \operatorname{MTD}\left(\theta_{m}\right) \mid m=2, \ldots, M\right\} .
$$

The dichotomized response model might be considered as a special case of the polychotomous response model, where $M=2, Y \in\{0,1\}$ (to provide the notation consistency, we use $\{0,1\}$ instead of $\{1,2\})$ and $m=1$, which is actually a 2-point ordinal toxicity grade system. The overall $\operatorname{MTD},{ }^{2} \operatorname{MTD}(\theta)$ is equal to the level-1 $\operatorname{MTD},{ }_{1} \operatorname{MTD}(\theta)=$ $\sup \{x \mid P(Y=1 \mid x) \leq \theta\}=\sup \{x \mid \psi(x, a) \leq \theta\}$, where $\psi(x, a)$ is defined as in the original CRM paper by O'Quigley et al. [6].

\section{THE CUMULATIVE PROBIT MODEL WITH LATENT VARIABLES}

To make the problem more interesting, we will assign a model structure on the $M$-point ordinal toxicity grade system, $\{\mathbb{Y}, \boldsymbol{P}, \boldsymbol{\theta}\}$, in the following context. Define the cumulative probabilities of the toxicity response $Y$ at dose $x$ as

$$
\eta_{y}(x)=P(Y \leq y \mid x)=\sum_{j=1}^{y} p_{j}(x), \quad y=1, \ldots, M-1 .
$$

Then, (1) is equivalent to

$$
{ }_{m} \operatorname{MTD}(\theta)=\sup \left\{x \mid \eta_{m-1}(x) \geq 1-\theta\right\} .
$$

Instead of assigning a model structure on toxicity response $Y$ directly, we will assign a model structure on the cumulative probabilities $\eta_{y}(x)$, which is the usual way to fit the ordered categorical data. Assume that there exists a latent continuous random variable $Z_{x}$ with probability density function $f_{Z_{x}}(z \mid x, \boldsymbol{\beta})$ or cumulative density function $F_{Z_{x}}(z \mid x, \boldsymbol{\beta})$, at dosage $x$, where $\boldsymbol{\beta}$ is the model parameters. Suppose that we observe toxicity grade $Y=y, y \in\{1,2, \ldots, m\}$, at dose $x$, where $Y=y \mid x$ if $\gamma_{y-1}<Z_{x} \leq \gamma_{y}$. Here, $\gamma_{0}, \gamma_{1}, \ldots, \gamma_{M-1}, \gamma_{M}$ are unknown bin boundaries (we define $\gamma_{0}=-\infty$ and $\left.\gamma_{M}=\infty\right)$. Hence,

$$
p_{y}(x)=P(Y=y \mid x)=F_{Z_{x}}\left(\gamma_{y} \mid x, \boldsymbol{\beta}\right)-F_{Z_{x}}\left(\gamma_{y-1} \mid x, \boldsymbol{\beta}\right)
$$

and the cumulative probabilities at dose $x$ is

$$
\begin{aligned}
\eta_{y}(x) & =P(Y \leq y \mid x)=\sum_{j=1}^{y} p_{j}(x) \\
& =\sum_{j=1}^{y}\left[F_{Z_{x}}\left(\gamma_{j} \mid x, \boldsymbol{\beta}\right)-F_{Z_{x}}\left(\gamma_{j-1} \mid x, \boldsymbol{\beta}\right)\right] \\
& =F_{Z_{x}}\left(\gamma_{y} \mid x, \boldsymbol{\beta}\right) .
\end{aligned}
$$

Therefore, (5) can be modified as

$$
{ }_{m} \operatorname{MTD}(\theta)=\sup \left\{x \mid F_{Z_{x}}\left(\gamma_{m-1} \mid x, \boldsymbol{\beta}\right) \geq 1-\theta\right\} .
$$

Given the critical value $m \in\{1,2, \ldots, M\}, Y \geq m$ implies that there is a level- $m$ severe toxicity at dosage $x$. If we define the probability of toxicity as

$$
\psi\left(x, \boldsymbol{\beta}, \gamma_{m-1}\right)=P(Y \geq m \mid x)=1-F_{Z_{x}}\left(\gamma_{m-1} \mid x, \boldsymbol{\beta}\right),
$$

then (1) becomes

$$
{ }_{m} \operatorname{MTD}(\theta)=\sup \left\{x \mid \psi\left(x, \boldsymbol{\beta}, \gamma_{m-1}\right) \leq \theta\right\},
$$

which is similar to the dichotomized response case. Furthermore, suppose we choose the normal latent variable, $Z_{x} \sim N\left(\mathbf{x}^{T} \boldsymbol{\beta}, 1\right)$, where $\boldsymbol{\beta}=\left(\beta_{0}, \beta_{1}\right)^{T}$ and $\mathbf{x}=(1, x)^{T}$, then, the probability of toxicity is

$$
\begin{aligned}
\psi\left(x, \boldsymbol{\beta}, \gamma_{m-1}\right) & =1-\Phi\left(\gamma_{m-1}-\mathbf{x}^{T} \boldsymbol{\beta}\right) \\
& =\Phi\left(\mathbf{x}^{T} \boldsymbol{\beta}-\gamma_{m-1}\right),
\end{aligned}
$$

for $m=1, \ldots, M-1$. Consequently, under the normal assumption, the MTD derived from (8) is

$$
{ }_{m} \operatorname{MTD}(\theta)=\frac{\gamma_{m-1}-\beta_{0}-\Phi^{-1}(1-\theta)}{\beta_{1}} .
$$

For an $M$-point ordinal toxicity grade system, $\left\{p_{1}(x), \ldots, p_{M}(x)\right\}$, associated with its target toxicity probability $\boldsymbol{\theta}=\left(\theta_{1}, \theta_{2}, \ldots, \theta_{M}\right)$, if the normal latent variable is used, then the overall MTD is defined as

$$
\begin{aligned}
& { }^{M} \operatorname{MTD}(\boldsymbol{\theta}) \\
& =\min \left\{\frac{\gamma_{m-1}-\beta_{0}-\Phi^{-1}\left(1-\theta_{m}\right)}{\beta_{1}} \mid m=2, \ldots, M\right\}
\end{aligned}
$$

In the next section, we will use the Bayes method to fit model (9). 


\section{THE LIKELIHOOD FUNCTION AND THE POSTERIOR DISTRIBUTION}

Denoted by $\mathscr{F}_{j}=\left\{\left(x_{1}, y_{1}\right), \ldots,\left(x_{j}, y_{j}\right)\right\}$ the history of the first $j$ assignments and responses, where $x_{l}$ is the dose level of the $l$ th patient (that is $x_{l} \in\left\{d_{1}, \ldots, d_{K}\right\}$ ) and $y_{l}$ is the observed response which takes one of $M$ ordered categories, $1, \ldots, M$ and $l=1, \ldots, j$. In model (9) of Section 3, both the regression parameter $\boldsymbol{\beta}$ and the bin boundary $\gamma=\left(\gamma_{1}, \gamma_{2}, \ldots, \gamma_{M-1}\right)$ are unknown. To ensure that the parameters are identifiable, it is necessary to impose one restriction on the bin boundary $\gamma$ (see Albert and Chib [1]). Without loss of generality, we take $\gamma_{1}=0$. The likelihood function of $\boldsymbol{\beta}$ and $\boldsymbol{\gamma}$ is

$$
\begin{aligned}
& L\left(\boldsymbol{\beta}, \boldsymbol{\gamma} \mid \mathscr{F}_{j}\right) \\
& \quad \propto \prod_{l=1}^{j} \sum_{y=1}^{M} I\left(y_{l}=y\right)\left[\Phi\left(\gamma_{y}-\mathbf{x}_{l}^{T} \boldsymbol{\beta}\right)-\Phi\left(\gamma_{y-1}-\mathbf{x}_{l}^{T} \boldsymbol{\beta}\right)\right] .
\end{aligned}
$$

It is straightforward to find the maximum likelihood estimate of $(\boldsymbol{\beta}, \boldsymbol{\gamma})$ by using the Newton-Raphson method and to obtain the approximate standard deviations of $(\boldsymbol{\beta}, \boldsymbol{\gamma})$ by using the second derivative of $\log$ likelihood evaluated at the maximum likelihood estimate. But, unfortunately, due to the small sample size in phase I clinical trials the accuracy of the MLE is questionable (see Albert and Chib [1]).

In order to increase the accuracy of the estimation, the Gibbs sampling algorithm (see Gelfand and Smith [5]) for the polychotomized response described in [1] can be generalized to this situation. We introduce $j$ latent variables $Z_{1}, Z_{2}, \ldots, Z_{j}$, where $Z_{l}$ are independent $N\left(\beta_{0}+\beta_{1} x_{l}, 1\right)$ and $x_{l}$ is the dose level of the $l$ th patient.

Define

$$
Y_{l}=y, \text { if } \gamma_{y-1}<Z_{l} \leq \gamma_{y}, \text { for } y=1,2, \ldots, M .
$$

The joint likelihood function of $(\boldsymbol{\beta}, \boldsymbol{\gamma}, \mathbf{Z})$ is

$$
\begin{aligned}
& L\left(\boldsymbol{\beta}, \boldsymbol{\gamma}, \mathbf{Z} \mid \mathscr{F}_{j}\right) \\
& \quad \propto \prod_{l=1}^{j}\left[\sum_{y=1}^{M} I\left(y_{l}=y\right) I_{\left(\gamma_{y-1}, \gamma_{y}\right)}\left(Z_{l}\right)\right] \phi\left(Z_{l} ; \mathbf{x}_{l}^{T} \boldsymbol{\beta}, 1\right) .
\end{aligned}
$$

Let $\pi(\boldsymbol{\beta}, \boldsymbol{\gamma})=\pi(\boldsymbol{\beta}) \pi(\boldsymbol{\gamma})$ be the prior on $(\boldsymbol{\beta}, \boldsymbol{\gamma})$, the joint posterior density function of $(\boldsymbol{\beta}, \boldsymbol{\gamma}, \mathbf{Z})$ given data $\mathscr{F}_{j}$ is

$$
\begin{aligned}
& \pi\left(\boldsymbol{\beta}, \boldsymbol{\gamma}, \mathbf{Z} \mid \mathscr{F}_{j}\right) \propto \pi(\boldsymbol{\beta}) \pi(\boldsymbol{\gamma}) \\
& \quad \times \prod_{l=1}^{j}\left[\sum_{y=1}^{M} I\left(y_{l}=y\right) I_{\left(\gamma_{y-1}, \gamma_{y}\right)}\left(Z_{l}\right)\right] \phi\left(Z_{l} ; \mathbf{x}_{l}^{T} \boldsymbol{\beta}, 1\right) .
\end{aligned}
$$

Since the probability of DLT is assumed to be increasing in dose level $x$ and the categories of toxicity grade are ordered, we need certain constraints on the prior distribution of $(\boldsymbol{\beta}, \boldsymbol{\gamma})$. The prior $\pi(\boldsymbol{\beta}, \boldsymbol{\gamma})$ should be defined on $\left\{(\boldsymbol{\beta}, \gamma) \mid \beta_{1}>0,-\infty<\gamma_{1}<\gamma_{2}<\cdots<\gamma_{M-1}<\infty\right\}$.
In order to evaluate (9) at any given dose $x \in$ $\left\{d_{1}, \ldots, d_{K}\right\}$, parameters $\left(\boldsymbol{\beta}, \gamma_{m-1}\right)$ need to be jointly generated from (11). Note that this joint posterior distribution (11) is complicated in the sense that it is difficult to normalize and sample from it directly. But computation of the marginal posterior distribution of $\left(\boldsymbol{\beta}, \gamma_{m-1}\right)$ using the Gibbs sampling algorithm requires only the posterior distribution of $\boldsymbol{\beta}$ conditional on $(\mathbf{Z}, \boldsymbol{\gamma})$, posterior distribution of $\mathbf{Z}$ conditional on $(\boldsymbol{\beta}, \boldsymbol{\gamma})$ and the posterior distribution of $\gamma$ conditional on $(\boldsymbol{\beta}, \mathbf{Z})$, and these full conditional posterior distributions are easy to obtain and easy to sample.

Based on (11), the full conditional posterior distributions are found as follows.

- $\pi\left(\boldsymbol{\beta} \mid \boldsymbol{\gamma}, \mathbf{Z}, \mathscr{F}_{j}\right)$

The posterior densities of $\beta_{0}$ and $\beta_{1}$, given $\gamma$ and $\mathbf{Z}$, is given by the following. If a flat prior $\pi\left(\beta_{0}\right) \propto 1$ is assigned on $\beta_{0}$, then,

$$
\beta_{0} \mid \beta_{1}, \mathbf{Z}, \mathscr{F}_{j} \sim N\left(\frac{\sum_{l=1}^{j}\left(z_{l}-\beta_{1} x_{l}\right)}{j}, \frac{1}{j}\right) .
$$

If a proper conjugate prior $N\left(\bar{\beta}_{0}, \bar{\sigma}_{0}^{2}\right)$ is assigned, then,

$$
\begin{aligned}
& \beta_{0} \mid \beta_{1}, \mathbf{Z}, \mathscr{F}_{j} \\
& \quad \sim N\left(\frac{\bar{\sigma}_{0}^{2} \sum_{l=1}^{j}\left(z_{l}-\beta_{1} x_{l}\right)+\bar{\beta}_{0}}{1+\bar{\sigma}_{0}^{2} j}, \frac{\bar{\sigma}_{0}^{2}}{1+\bar{\sigma}_{0}^{2} j}\right) .
\end{aligned}
$$

If a flat prior $\pi\left(\beta_{1}\right) \propto I\left(\beta_{1}>0\right)$ is assigned, then,

$$
\beta_{1} \mid \beta_{0}, \mathbf{Z}, \mathscr{F}_{j} \sim N\left(\frac{\sum_{l=1}^{j}\left(z_{l}-\beta_{0}\right) x_{l}}{\sum_{l=1}^{j} x_{l}^{2}}, \frac{1}{\sum_{l=1}^{j} x_{l}^{2}}\right),
$$

for $\beta_{1}>0$. If a proper conjugate truncated normal prior $N\left(\bar{\beta}_{1}, \bar{\sigma}_{1}^{2}\right) I\left(\beta_{1}>0\right)$ is assigned, then,

$$
\begin{aligned}
& \beta_{1} \mid \beta_{0}, \mathbf{Z}, \mathscr{F}_{j} \\
& \quad \sim N\left(\frac{\bar{\sigma}_{1}^{2} \sum_{l=1}^{j}\left(z_{l}-\beta_{0}\right) x_{l}+\bar{\beta}_{1}}{1+\bar{\sigma}_{1}^{2} \sum_{l=1}^{j} x_{l}^{2}}, \frac{\bar{\sigma}_{1}^{2}}{1+\bar{\sigma}_{1}^{2} \sum_{l=1}^{j} x_{l}^{2}}\right),
\end{aligned}
$$

for $\beta_{1}>0$. If a proper, but non-conjugate exponential prior $\exp \left\{-\beta_{1}\right\} I\left(\beta_{1}>0\right)$ is assigned, then,

$$
\beta_{1} \mid \beta_{0}, \mathbf{Z}, \mathscr{F}_{j} \sim N\left(\frac{\sum_{l=1}^{j}\left(z_{l}-\beta_{0}\right) x_{l}-1}{\sum_{l=1}^{j} x_{l}^{2}}, \frac{1}{\sum_{l=1}^{j} x_{l}^{2}}\right),
$$

for $\beta_{1}>0$.

$$
\text { - } \pi\left(\mathbf{Z} \mid \boldsymbol{\beta}, \boldsymbol{\gamma}, \mathscr{F}_{j}\right)
$$

$Z_{l}$ 's are latent variables, not parameters. Hence no priors are needed. Using (11), the posterior density of $\mathbf{Z}$, given $\boldsymbol{\beta}$ and $\gamma$, is 
(17)

$$
\begin{aligned}
& \pi\left(\mathbf{Z} \mid \boldsymbol{\beta}, \boldsymbol{\gamma}, \mathscr{F}_{j}\right) \\
& \quad \propto \prod_{l=1}^{j}\left[\sum_{y=1}^{M} I\left(y_{l}=y\right) I_{\left(\gamma_{y-1}, \gamma_{y}\right)}\left(Z_{l}\right)\right] \phi\left(Z_{l} ; \mathbf{x}_{l}^{T} \boldsymbol{\beta}, 1\right),
\end{aligned}
$$

which implies that $Z_{1}, Z_{2}, \ldots, Z_{j}$ are independent with

$$
Z_{l} \mid \boldsymbol{\beta}, \boldsymbol{\gamma}, \mathscr{F}_{j} \sim N\left(\mathbf{x}_{l}^{T} \boldsymbol{\beta}, 1\right) I\left(\gamma_{y-1}<Z_{l}<\gamma_{y}\right),
$$

for $y_{l}=y$, where $y$ takes one of the $M$ ordered categories, $1, \ldots, M$ and $l=1, \ldots, j-1$. (18) implies that $Z_{l} \mid \boldsymbol{\beta}, \boldsymbol{\gamma}, \mathscr{F}_{j}$ is a normal random variable truncated at the left $\gamma_{y-1}$ and right $\gamma_{y}$ when $y_{l}=y$.

$$
\text { - } \pi\left(\boldsymbol{\gamma} \mid \boldsymbol{\beta}, \mathbf{Z}, \mathscr{F}_{j}\right)
$$

According to (11), the posterior density of $\boldsymbol{\gamma}$, given $\boldsymbol{\beta}$ and $\mathbf{Z}$, is

$$
\begin{aligned}
& \pi\left(\boldsymbol{\gamma} \mid \boldsymbol{\beta}, \mathbf{Z}, \mathscr{F}_{j}\right) \\
& \quad \propto \pi(\boldsymbol{\gamma}) \prod_{l=1}^{j}\left[\sum_{y=1}^{M} I\left(y_{l}=y\right) I_{\left(\gamma_{y-1}, \gamma_{y}\right)}\left(Z_{l}\right)\right],
\end{aligned}
$$

which implies that $\gamma_{1}, \gamma_{2}, \ldots, \gamma_{j}$ are dependent. Suppose a flat prior is assigned on $\gamma$

$$
\pi(\gamma)=I\left(-\infty<\gamma_{1}<\gamma_{2}<\cdots<\gamma_{M-1}<\infty\right),
$$

then the full conditional posterior distribution of $\gamma_{i}$ given $\boldsymbol{\beta}, \boldsymbol{\gamma}_{-i}=\left\{\gamma_{y}, y \neq i\right\}, \mathbf{Z}$ and $\mathscr{F}_{j}$, is

$$
\begin{aligned}
& \pi\left(\gamma_{i} \mid \boldsymbol{\beta}, \boldsymbol{\gamma}_{-i}, \mathbf{Z}, \mathscr{F}_{j}\right) \\
& \propto \\
& \quad \prod_{l=1}^{j}\left[I\left(y_{l}=i\right) I_{\left(\gamma_{i-1}, \gamma_{i}\right)}\left(Z_{l}\right)\right. \\
& \left.\quad+I\left(y_{l}=i+1\right) I_{\left(\gamma_{i}, \gamma_{i+1}\right)}\left(Z_{l}\right)\right] .
\end{aligned}
$$

This conditional distribution can be seen to be uniform, i.e.,

$$
\gamma_{i} \mid \boldsymbol{\beta}, \boldsymbol{\gamma}_{-i}, \mathbf{Z}, \mathscr{F}_{j} \sim \mathrm{U}(a, b),
$$

where $a=\max \left\{\max \left\{Z_{l}: y_{l}=i\right\}, \gamma_{i-1}\right\}$ and $b=$ $\min \left\{\min \left\{Z_{l}: y_{l}=i+1\right\}, \gamma_{i+1}\right\}$.

Based on the Gibbs sampler, $\beta_{0}, \beta_{1}, \mathbf{Z}$ and $\gamma$ could be generated from those full conditional posterior distributions.

After drawing from the marginal joint posterior distribution $\pi\left(\boldsymbol{\beta}, \gamma_{m-1}\right)$, one can estimate the overall MTD by using the following formula.

$$
\begin{aligned}
{ }^{M} & \widehat{\operatorname{MTD}}(\boldsymbol{\theta}) \\
= & \min \left\{\frac{E^{\pi\left(\gamma_{m-1} \mid \mathscr{F}_{j}\right)} \gamma_{m-1}-E^{\pi\left(\beta_{0} \mid \mathscr{F}_{j}\right)} \beta_{0}}{E^{\pi\left(\beta_{1} \mid \mathscr{F}_{j}\right)} \beta_{1}}\right. \\
& \left.-\frac{\Phi^{-1}\left(1-\theta_{m}\right)}{E^{\pi\left(\beta_{1} \mid \mathscr{F}_{j}\right)} \beta_{1}} \mid m=2, \ldots, M\right\} .
\end{aligned}
$$

The estimates of the expectations in (21) are obtained based on the simulation. Suppose one has obtained $N$ generations of $\left(\beta_{0}, \beta_{1}, \gamma_{m-1}\right)$, which are $\left(\beta_{0}^{(i)}, \beta_{1}^{(i)}, \gamma_{m-1}^{(i)}\right), i=$ $1,2, \ldots, N$, then,

$$
\begin{aligned}
& \hat{E}^{\pi\left(\beta_{0} \mid \mathscr{F}_{j}\right)}\left(\beta_{0}\right)=\frac{1}{N} \sum_{i=1}^{N} \beta_{0}^{(i)}, \\
& \hat{E}^{\pi\left(\beta_{1} \mid \mathscr{F}_{j}\right)}\left(\beta_{1}\right)=\frac{1}{N} \sum_{i=1}^{N} \beta_{1}^{(i)}, \\
& \hat{E}^{\pi\left(\gamma_{m-1} \mid \mathscr{F}_{j}\right)}\left(\gamma_{m-1}\right)=\frac{1}{N} \gamma_{m-1}^{(i)} .
\end{aligned}
$$

The formula in (21) does not guarantee to yield an exact dose level as one of the predetermined dose levels. More often we choose the dose that is closest to the computed result.

\section{SIMULATION RESULTS}

To evaluate the operating characteristics of the polychotomous response model, a simulation study is performed. We use a 5-point ordinal toxicity scale, i.e., the polychotomous toxicity response $Y$ takes one of the 5 values, $\{1, \ldots, 5\}$ at any given dose level, with grade 1 representing no toxicity, grade 2 minor toxicity, grade 3 moderate toxicity, grade 4 severe toxicity, and grade 5 very severe or life threatening toxicity. We suppose there are six ordered dose levels, $x_{1}, x_{2}, \ldots, x_{6}$ and the data are simulated according to the following probabilities,

$$
p_{i j}=P\left(Y=i \mid \text { Dose }=x_{j}\right),
$$

for $i=1, \ldots, 5$, and $j=1, \ldots, 6$, where $\sum_{i=1}^{5} p_{i j}=1$ for any $j=1, \ldots, 6$, and, for any $i_{0}=2, \ldots, 5, \sum_{i=i_{0}}^{5} p_{i j^{\prime}}<$ $\sum_{i=i_{0}}^{5} p_{i j^{\prime \prime}}$, for any $1 \leq j^{\prime}<j^{\prime \prime} \leq 6$. Table 1 shows the true probabilities of each grade (1-5) at each dose level (1-6) for four simulation scenarios.

In this simulation study, the probabilities are generated in one of the following two ways.

(1) Normal latent: $p_{i j}=P\left(\gamma_{j-1}<Z_{i} \leq \gamma_{j}\right)$, for $i=$ $1, \ldots, 5$ and $j=1, \ldots, 6$, where $Z_{i} \sim N\left(\beta_{0}+\beta x_{i}, 1\right), \gamma_{0}=$ $-\infty, \gamma_{5}=\infty$ and $\boldsymbol{\beta}=\left(\beta_{0}, \beta_{1}\right), \gamma=\left(\gamma_{1}, \ldots, \gamma_{4}\right)$ are set to different values for different scenarios.

(2) General situation: for any $i_{0}=2, \ldots, 5, \sum_{i=i_{0}}^{5} p_{i j^{\prime}}<$ $\sum_{i=i_{0}}^{5} p_{i j^{\prime \prime}}$, if $1 \leq j^{\prime}<j^{\prime \prime} \leq 6$.

The target toxicity probabilities, $\boldsymbol{\theta}=\left(\theta_{1}, \ldots, \theta_{5}\right)$, are set in three different combinations. For each scenario we use 200 duplications (trials). In each trial, we recruit 30 patients in 10 cohorts with 3 patients per cohort. The first cohort of subjects in each trial was treated at the lowest dose. Additional constraints follow those of Faries [4] where dose escalation was limited to a maximum of 1 dose between consecutive subjects. The dose closest to the final updated 
Table 1. True probabilities of toxicity for various grade and dose levels

\begin{tabular}{cccccccc}
\hline \hline Scenario & Grade & \multicolumn{7}{c}{ Dose level } \\
\cline { 3 - 8 } & & $x_{1}$ & $x_{2}$ & $x_{3}$ & $x_{4}$ & $x_{5}$ & $x_{6}$ \\
\hline \multirow{2}{*}{$\mathrm{A}$} & 1 & 0.87 & 0.78 & 0.70 & 0.51 & 0.19 & 0.12 \\
& 2 & 0.08 & 0.12 & 0.15 & 0.19 & 0.16 & 0.13 \\
& 3 & 0.03 & 0.06 & 0.09 & 0.15 & 0.20 & 0.18 \\
& 4 & 0.01 & 0.03 & 0.04 & 0.09 & 0.18 & 0.20 \\
& 5 & 0.00 & 0.01 & 0.02 & 0.06 & 0.27 & 0.37 \\
\hline \multirow{2}{*}{$\mathrm{B}$} & 1 & 0.87 & 0.78 & 0.70 & 0.51 & 0.19 & 0.12 \\
& 2 & 0.08 & 0.12 & 0.15 & 0.19 & 0.16 & 0.13 \\
& 3 & 0.02 & 0.04 & 0.05 & 0.08 & 0.10 & 0.09 \\
& 4 & 0.02 & 0.04 & 0.06 & 0.12 & 0.20 & 0.19 \\
& 5 & 0.01 & 0.02 & 0.04 & 0.10 & 0.36 & 0.47 \\
\hline \multirow{2}{*}{$\mathrm{C}$} & 1 & 0.82 & 0.73 & 0.53 & 0.35 & 0.23 & 0.15 \\
& 2 & 0.10 & 0.14 & 0.19 & 0.20 & 0.17 & 0.15 \\
& 3 & 0.05 & 0.08 & 0.14 & 0.18 & 0.20 & 0.19 \\
& 4 & 0.02 & 0.04 & 0.08 & 0.14 & 0.17 & 0.19 \\
& 5 & 0.01 & 0.02 & 0.06 & 0.13 & 0.23 & 0.32 \\
\hline \multirow{2}{*}{$\mathrm{D}$} & 1 & 0.83 & 0.75 & 0.55 & 0.37 & 0.24 & 0.16 \\
& 2 & 0.09 & 0.12 & 0.17 & 0.18 & 0.16 & 0.14 \\
& 3 & 0.03 & 0.05 & 0.08 & 0.10 & 0.10 & 0.09 \\
& 4 & 0.03 & 0.05 & 0.11 & 0.16 & 0.19 & 0.20 \\
& 5 & 0.02 & 0.03 & 0.09 & 0.19 & 0.31 & 0.41 \\
\hline & & & & & & &
\end{tabular}

MTD was taken to be the final recommended dose. The updated MTD was then calculated by using (21).

The working model is

$$
\left\{\begin{array}{c}
Y=y \mid x, \text { if } \gamma_{y-1}<Z_{x} \leq \gamma_{y}, \text { where } y=1,2, \ldots, 5, \\
\text { and, }-\infty=\gamma_{0}<\gamma_{1}<\cdots<\gamma_{4}<\gamma_{5}=\infty, \\
Z_{x} \sim N\left(\beta_{0}+\beta_{1} x, 1\right), \text { for } x \in\left\{x_{1}, x_{2}, \ldots, x_{6}\right\} .
\end{array}\right.
$$

We always set a prior $\pi\left(\beta_{0}, \beta_{1}\right) \propto \exp \left(-\beta_{1}\right), \beta_{1}>0$ on the parameter in each scenario. To ensure that the parameters are identifiable, it is necessary to impose one restriction on the bin boundary $\gamma=\left(\gamma_{1}, \ldots, \gamma_{4}\right)$. Since toxicity grades 3 , 4 and 5 represent moderate, severe and very severe or life threatening toxicity, we take $\gamma_{2}=0$. Table 2 is an illustration of the process from one of the simulated trials. We frame those toxicity grades which are grade 3,4 and 5 , since they represent the moderate, severe and very severe or life threatening toxicities.

Figure 4 shows an example of the simulated parameters of $\boldsymbol{\beta}=\left(\beta_{0}, \beta_{1}\right)$ and $\boldsymbol{\gamma}=\left(\gamma_{1}, \gamma_{3}, \gamma_{4}\right)$ at the sample size 30 . Based on the trace in Figure 4, the MCMC results converge and based on the density in Figure 4, all the constraints on the model parameters, such as $\beta_{1}>0, \gamma_{1}<0, \gamma_{3}>0$ and $\gamma_{4}>0$, are satisfied.

Table 3 shows the simulation results. In Table 3 , we only display target toxicity probabilities $\left(\theta_{3}, \theta_{4}, \theta_{5}\right)$, since
Table 2. An illustration of one of the simulated trials

\begin{tabular}{ccccc}
\hline \hline Cohort & Dose level & \multicolumn{3}{c}{ Toxicity grade } \\
\cline { 3 - 5 } & & Subject 1 & Subject 2 & Subject 3 \\
\hline 1 & $x_{1}$ & 2 & 2 & 1 \\
2 & $x_{2}$ & 1 & 1 & 1 \\
3 & $x_{3}$ & 1 & 1 & 3 \\
4 & $x_{4}$ & 5 & 5 & 1 \\
5 & $x_{5}$ & 1 & 1 & 4 \\
6 & $x_{4}$ & 1 & 1 & 1 \\
7 & $x_{3}$ & 1 & 1 & 4 \\
8 & $x_{4}$ & 1 & 2 & 2 \\
9 & $x_{4}$ & 3 & 1 & 2 \\
10 & $x_{4}$ & & & \\
\hline
\end{tabular}

Decision $\quad x_{4}$

$\theta_{1}$ has been always set to be equal to 1 and $\theta_{2}$ is associated with toxicity grade 2 (which is minor toxicity) and above, hence we also set $\theta_{2}=1$. For $\left(\theta_{3}, \theta_{4}, \theta_{5}\right)$, we consider three settings, $(0.3,0.3,0.3)$ which is equivalent to the dichotomized model, $(0.3,0.1,0.05)$ which indicates that toxicity grades 4 and 5 are considered and differentiated, $(0.3,0.06,0.02)$ which indicates that grades 4 and 5 are more severe in toxicity than grade 3 . Table 3 shows that both percent of recommended level and percent of patient allocation are toward the lower dose levels in general. For the first scenario, $83 \%$ of the recommendations are $x_{4}$ for dichotomized model. However, for the polychotomous model, $54 \%$ and $21 \%$ of the recommendations are $x_{4}$ for target toxicity probability settings $(0.3,0.1,0.05)$ and $(0.3,0.06,0.02)$, respectively. In addition, for $(0.3,0.06,0.02), 46 \%$ of the recommendations are $x_{3}$, which is the largest recommendation rate among all six dose levels. As for patient allocation, $34 \%$ and $20.4 \%$ of them allocate to $x_{4}$ for $(0.3,0.1,0.05)$ and $(0.3,0.06,0.02)$, respectively (decrease from $44.6 \%$ for dichotomized model). Similar results are shown for the scenarios B, C and D. In conclusion, the polychotomous model, which takes a severity level of toxicity into account, reduces the number of patients allocated to the higher toxicity dose level. It also reduces the risk of toxicity for patients in the phase I study.

\section{CONCLUSIONS AND DISCUSSIONS}

In this study, we propose a new definition of the overall $\operatorname{MTD},{ }^{M} \operatorname{MTD}(\boldsymbol{\theta})$, in the case of the polychotomous toxicity responses and the analytic properties of the overall MTD are also examined. It is shown that the traditional definition of MTD in the case of the dichotomous (binary) toxicity responses is a special case of the overall MTD. In order to find the overall MTD, ${ }^{M} \operatorname{MTD}(\boldsymbol{\theta})$, in practice, the target toxicity probability $\boldsymbol{\theta}=\left\{\theta_{1}, \ldots, \theta_{M}\right\}$, where $\theta_{m}$ is the target toxicity probability corresponding to the level- $m$ severe 
Trace
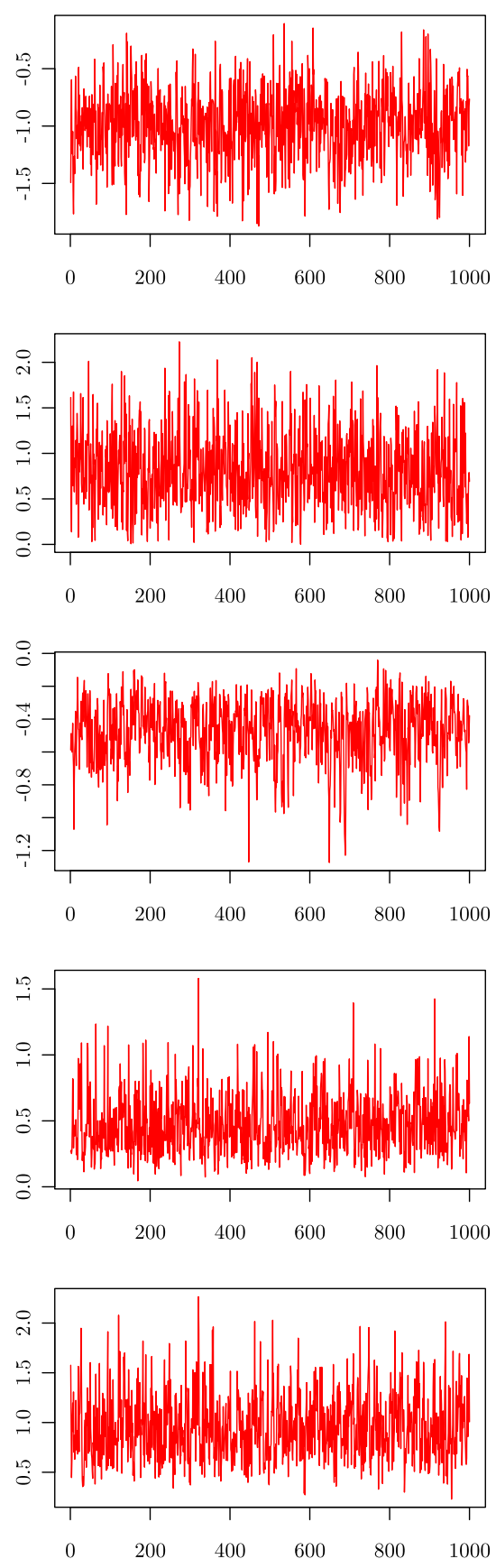

$\mathrm{N}=1000$
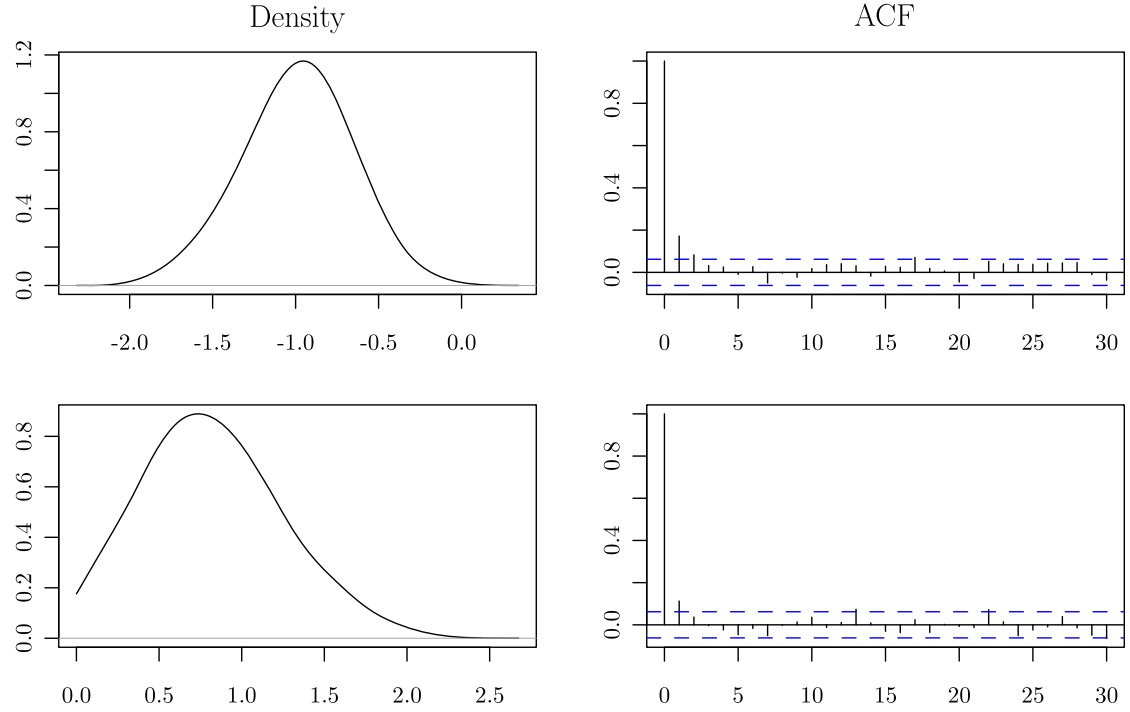

$\beta_{1}$
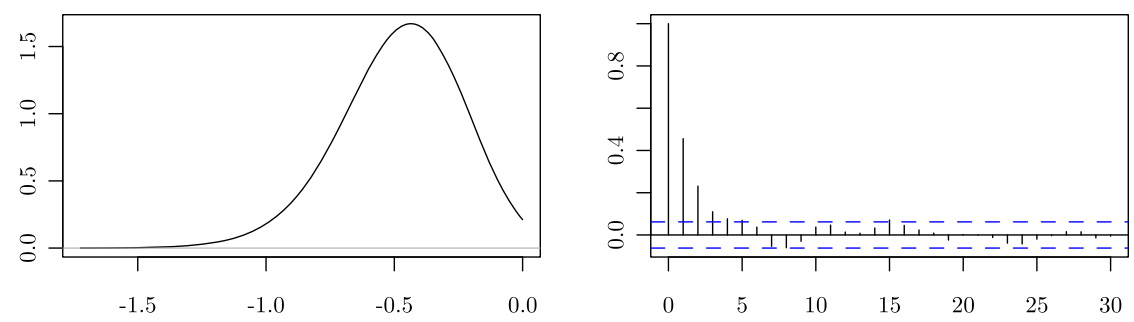

$\gamma_{1}$
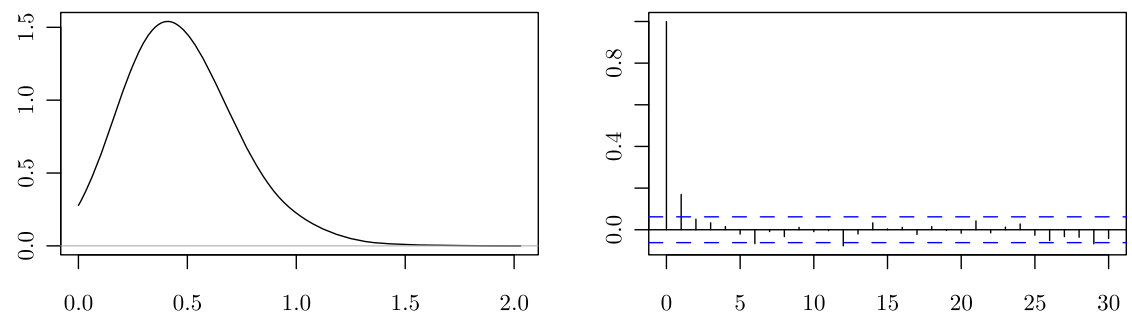

$\gamma_{3}$
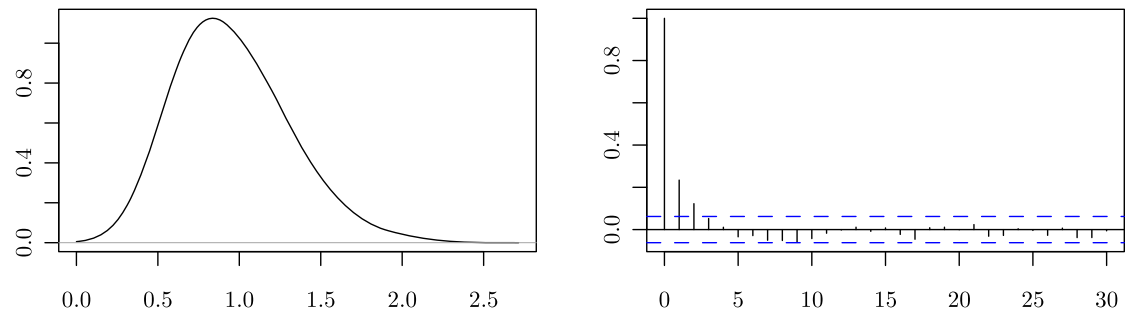

$\gamma_{4}$

Lag

Figure 4. An illustration of simulated $\boldsymbol{\beta}$ and $\boldsymbol{\gamma}($ sample size $=30)$. The trace column gives the time series plot for each parameter. After burn-in the first 1,000 generations and thinning in each 100 generations, the time series plots appear to be stationary. The ACF column gives the autocorrelation function for each parameter and it shows that the parameters are not significantly autocorrelated. From the density column, it is clear that $\beta_{1}>0, \gamma_{1}<0, \gamma_{3}>0$ and $\gamma_{4}>0$, which satisfy the constraints on the model parameters.

toxicity $T_{m}, m \in\{1,2, \ldots, M\}$, needs to be pre-specified. The determination of each $\theta_{m}, m=1, \ldots, M$, is same as the determination of the target probability $\theta$ in the dichoto- mous (binary) toxicity case. Therefore, compared with the existing methods described in $[3,8,11]$, there is not much more effort needed to interact with the physicians. 
Table 3. Simulation results

\begin{tabular}{|c|c|c|c|c|c|c|c|c|}
\hline \multirow[t]{2}{*}{ Scenario } & \multicolumn{2}{|l|}{ Target } & \multicolumn{6}{|c|}{ Dose level } \\
\hline & $\left(\begin{array}{lll}\left(\theta_{3},\right. & \theta_{4}, & \theta_{5}\end{array}\right)$ & & $x_{1}$ & $x_{2}$ & $x_{3}$ & $x_{4}$ & $x_{5}$ & $\overline{x_{6}}$ \\
\hline \multicolumn{9}{|l|}{$\mathrm{A}$} \\
\hline & $(.30, .30, .30)$ & Rec. & & 1.5 & 11.5 & $\underline{83.0}$ & 4.0 & \\
\hline & & Exp. & 12.1 & 14.5 & 21.8 & 44.6 & 6.7 & 0.2 \\
\hline & $(.30, .10, .05)$ & Rec. & 3.5 & 7.0 & 34.5 & $\underline{54.0}$ & 1.0 & \\
\hline & & Exp. & 16.5 & 16.8 & 27.7 & $\overline{34.0}$ & 4.9 & 0.1 \\
\hline & $(.30, .06, .02)$ & Rec. & 13.0 & 20.0 & $\underline{46.0}$ & 21.0 & & \\
\hline & & Exp. & 22.1 & 24.4 & $\overline{29.4}$ & 20.4 & 3.7 & \\
\hline \multicolumn{9}{|l|}{ B } \\
\hline & $(.30, .30, .30)$ & Rec. & & 0.5 & 13.0 & $\underline{80.5}$ & 6.0 & \\
\hline & & Exp. & 12.7 & 13.4 & 22.9 & 42.9 & 7.7 & 0.4 \\
\hline & $(.30, .10, .05)$ & Rec. & 6.5 & 18.5 & $\underline{47.0}$ & 27.5 & 0.5 & \\
\hline & & Exp. & 20.6 & 22.3 & 31.7 & 22.2 & 2.9 & 0.3 \\
\hline & $(.30, .06, .02)$ & Rec. & 28.0 & $\underline{33.5}$ & 32.6 & 6.0 & & \\
\hline & & Exp. & 33.0 & 26.9 & 26.4 & 11.6 & 1.9 & 0.1 \\
\hline \multicolumn{9}{|l|}{$\mathrm{C}$} \\
\hline & $(.30, .30, .30)$ & Rec. & 1.0 & 9.5 & $\underline{67.5}$ & 21.0 & 1.0 & \\
\hline & & Exp. & 14.4 & 21.6 & 42.3 & 18.0 & 3.5 & 0.2 \\
\hline & $(.30, .10, .05)$ & Rec. & 6.5 & 32.5 & $\underline{53.5}$ & 8.0 & & \\
\hline & & Exp. & 18.2 & 32.2 & $\overline{37.1}$ & 11.2 & 1.2 & 0.1 \\
\hline & $(.30, .06, .02)$ & Rec. & 20.5 & $\underline{49.5}$ & 29.0 & 1.0 & & \\
\hline & & Exp. & 28.2 & 37.3 & 27.1 & 6.3 & 1.1 & \\
\hline \multicolumn{9}{|l|}{$\mathrm{D}$} \\
\hline & $(.30, .30, .30)$ & Rec. & & 7.0 & $\underline{68.5}$ & 22.5 & 2.0 & \\
\hline & & Exp. & 14.1 & 21.8 & 42.8 & 17.6 & 3.4 & 0.4 \\
\hline & $(.30, .10, .05)$ & Rec. & 17.5 & $\underline{48.0}$ & 32.5 & 2.0 & & \\
\hline & & Exp. & 27.2 & 35.9 & 29.6 & 6.3 & 1.0 & \\
\hline & $(.30, .06, .02)$ & Rec. & 43.0 & $\underline{45.5}$ & 11.5 & & & \\
\hline & & Exp. & 41.3 & $\overline{34.8}$ & 19.1 & 4.1 & 0.7 & \\
\hline
\end{tabular}

As an illustration of our research, we utilize the cumulative probit model (9) with the normal latent variables as the working model. The simulation studies show that the cumulative probit model, which takes severity level of toxicity into account, reduces the number of patients allocated to the higher toxicity dose level. That reduces the risk of toxicity for patients in the phase I study. We also perform simulation studies on various settings of the target toxicity probability $\boldsymbol{\theta}=\left\{\theta_{1}, \ldots, \theta_{M}\right\}$. Simulation results shows that our method is robust in the change of the target toxicity probability.

In practice, other working models, such as the cumulative logistic model or other suitable models, can be utilized under the same framework. Consequently, solutions of the overall MTD, such as shown in (21), may have different forms.

In addition, prior elicitation often is an important task. In this study, we provide full conditional distributions for the parameters and latent variables for various priors. When more complex models or hard-to-deal-with priors are used, the difficulties might arise when complex full conditional distributions are obtained. However, many simulation methods can be applied to handle those difficulties, such as acceptance-rejection algorithms or the Metropolis-Hastings algorithm.

\section{ACKNOWLEDGEMENT}

The authors would like to thank the referee and the editors for their insightful comments and useful suggestions.

Received 27 September 2012

\section{REFERENCES}

[1] Albert, J. H., and Chib, S. (1993). Bayesian analysis of binary and polychotomous response data. Journal of the American Statistical Association 88 669-679. MR1224394

[2] Babb, J., Rogatko, A., and Zacks, S. (1998). Cancer phase I clinical trials: efficient dose escalation with overdose control. Statistics in Medicine 17 1103-1120.

[3] Bekele, B. N., and Thall, P. F. (2004). Dose-finding based on multiple toxicities in a soft tissue sarcoma trial. Journal of the American Statistical Association 99 26-35. MR2061885

[4] Faries, D. (1994). Practical modifications of the continual reassessment method for phase I cancer clinical trials. Journal of Biopharmaceutical Statistics 4 147-164. 
[5] Gelfand, A. E., and Smith, A. F. M. (1990). Sampling-based approaches to calculating marginal densities. Journal of the American Statistical Association 85 398-409. MR1141740

[6] O'Quigley, J., Pepe, M., and Fisher, L. (1990). Continual reassessment method: A practical design for phase I clinical trials in cancer. Biometrics 46 33-48. MR1059105

[7] Storer, B. E. (1989). Design and analysis of phase I clinical trials. Biometrics 45 925-937. MR1029610

[8] Wang, C., Chenb, T. T., and Tyanc, I. (2000). Designs for phase I cancer clinical trials with differentiation of graded toxicity. Communications in Statistics-Theory and Methods 29 975987.

[9] YANG, X., Ye, K., and WANG, Y. (2011). A study of the probit model with latent variables in phase I clinical trials. Working paper series, College of Business, University of Texas at San Antonio.

[10] YiN, G., and YuAN, Y. (2009). Bayesian model averaging continual reassessment method in phase I clinical trials. Journal of the American Statistical Association 104 954-968. MR2750228
[11] Yuan, Z., Chappell, R., and Bailey, H. (2007). The continual reassessment method for multiple toxicity grades: A Bayesian quasi-likelihood approach. Biometrics 63 173-179. MR2345586

\section{Xiaobin Yang}

Analytic Focus, LLC

4939 De Zavala Road

San Antonio, TX 78249

USA

E-mail address: zbc592@gmail.com

Keying Ye

One UTSA Circle

Department of Management Science and Statistics

University of Texas at San Antonio

San Antonio, TX 78249

USA

E-mail address: keying.ye@utsa.edu 